\title{
Commentary: 3D Laparoscopy-Assisted Operation to Adult Intussusceptions During Perioperative Period of Liver Transplantation: Case Report and Literature Review
}

\author{
Som P. Singh ${ }^{1 *}$, Kiera G. Borthwick ${ }^{2}$ and Fahad M. Qureshi ${ }^{1}$ \\ ${ }^{1}$ Department of Biomedical Sciences, University of Missouri-Kansas City School of Medicine, Kansas, MO, United States, \\ ${ }^{2}$ Department of Neurosciences, Washington and Lee University, Lexington, VA, United States
}

Keywords: 3D, laparoscopy, intussusceptions, liver transplant, outcomes

OPEN ACCESS

Edited by:

Bo Wang,

First Affiliated Hospital of X'ian Jiaotong University, China

Reviewed by:

Jose M. Ramia,

Servicio de Salud de Castilla La

Mancha, Spain

*Correspondence:

Som P. Singh

somsingh@mail.umkc.edu

Specialty section:

This article was submitted to

Visceral Surgery,

a section of the journal

Frontiers in Surgery

Received: 25 August 2021 Accepted: 24 September 2021

Published: 20 October 2021

Citation:

Singh SP, Borthwick KG and Qureshi FM (2021) Commentary: 3D Laparoscopy-Assisted Operation to

Adult Intussusceptions During

Perioperative Period of Liver Transplantation: Case Report and

Literature Review.

Front. Surg. 8:764741.

doi: 10.3389/fsurg.2021.764741

\section{A Commentary on:}

3D Laparoscopy-Assisted Operation to Adult Intussusceptions During Perioperative Period of Liver Transplantation: Case Report and Literature Review

by Gao, Q., Yuan, S., Li, Y., Chen, C., Liu, X., and Yu, J. (2021). Front. Surg. 8:647767. doi: $10.3389 /$ fsurg.2021.647767

In the case report by Gao et al. (1), a 32-year-old male presented with pain and distention in the lower left quadrant following a liver transplant for liver failure and cirrhosis. Initial CT scans showed proximal enterostenosis, but symptoms of constipation gradually became more severe. Another CT scan was completed 4 days later, and an exploratory laparoscopy was performed, indicating antegrade intussusception, where the proximal bowel invaginated itself into the distal bowel. Adhesion removal and a simple reduction were completed, and a small incision was made to ensure local adhesion restoration. The serosa was sutured integrally. Reported pain and distention decreased gradually following the procedure, and the patient was discharged 9 days later, with no symptoms at a 2 -month follow-up.

Laparoscopy is becoming a more favorable method for surgical treatment of intussusception and causes less tissue damage and immune responses compared to an open laparotomy (2). Moreover, laparoscopy decreases the duration of hospital stay and the time to first food intake (3). Post-operative complication rates are similar between laparotomy and laparoscopy, but the complications from laparoscopy are often more severe (i.e., perforation) (3).

Surgical options, including laparotomy or laparoscopy, remains the main treatment plan for adult intussusception, although the specifics of the interventions vary. Surgical interventions for intussusception tend to include one of the five following procedures: resection without reduction, reduction followed by resection, reduction only, enterotomy and mass excision, and negative exploration (4). Most studies support case-by-case determinations of whether reductions should be performed, while there is more limited support for resection without reduction. Resection without reduction helps prevent the potential risks of implantation metastasis in intussusception cases involving cancerous tumors. Overall, the best surgical method depends on the location of the intussusception. In enteric intussusception, reduction followed by resection is most successful. 
In colonic intussusception, resection alone is most effective. Ileocolic intussusception has an intermediate location and requires a more selective, combined approach (4).

There are also non-invasive and non-surgical treatments for intussusceptions, including hydrostatic, pneumatic, and gas enemas under ultrasound or fluoroscopy. In addition, colonoscopy has been considered as a treatment option. Unless there is an imminent indication for surgery, as in emergency cases, it is frequently recommended that diagnostic evaluation and more conservative, non-surgical treatment options be utilized, especially in pediatric populations $(4,5)$. Ultrasoundguided hydrostatic reductions can serve as a safer, non-operative treatment for intussusceptions (6). Hydrostatic reductions have similar safety and efficacy compared to pneumatic reduction, and ultrasound guided intussusception reduction should continue to be evaluated, as it does not require radiation exposure (7). Colonoscopy has also successfully reversed invaginations in twothirds of pediatric patients (8) and has also been effective in adult patients (9).

\section{FUTURE APPLICATIONS}

Laparoscopic augmented reality navigation, LARN, is a novel technique that has shown promise in many surgical procedures, including hepatectomy for liver cancer (10). LARN helps localize important features, like tumors and vessels, and has been shown to reduce intraoperative bleeding, blood transfusion rates, and postoperative recovery time (10). Augmented reality, AR, used with video see-through, gives real-time, three-dimensional anatomical visualization of a variety of structures, including nerves, vessels, lesions, and more. AR has been investigated in neurosurgery (11), otolaryngology (12), urologic laparoscopic surgery (13), and more. AR is more accepted and commonly used in surgeries like these, which are centered around rigid or semi-rigid structures (i.e., bones). Therefore, the use of LARN

\section{REFERENCES}

1. Gao Q, Yuan S, Li Y, Chen C, Liu X, Yu J. 3D laparoscopy-assisted operation to adult intussusceptions during perioperative period of liver transplantation: case report and literature review. Front Surg. (2021) 8:647767. doi: 10.3389/fsurg.2021.647767

2. Pedano N, Rotellar F, Alvarez-Cienfuegos J, Arredondo J, Bellver M, Martínez P, et al. Efficacy of laparoscopic approach in the management of early liver transplant complications. Transplant Proc. (2012) 44:1560-1. doi: 10.1016/j.transproceed.2012.05.024

3. Kang S, Lee SI, Min BW, Lee TH, Baek SJ, Kwak JM, et al. A multicentre comparative study between laparoscopic and open surgery for intussusception in adults. Colorectal Dis. (2020) 22:1415-21. doi: 10.1111/codi.15102

4. Hong KD, Kim J, Ji W, Wexner SD. Adult intussusception: A systematic review and meta-analysis. Tech Coloproctol. (2019) 23:315-24. doi: 10.1007/s10151-019-01980-5

5. Guillén-Paredes MP, Campillo-Soto A, Martín-Lorenzo JG, TorralbaMartínez JA, Mengual-Ballester M, Cases-Baldó MJ, et al. Adult intussusception: 14 case reports and their outcomes. Rev Española Enfermedades Digest. (2010) 102:5. doi: 10.4321/S1130-01082010000100005

6. Xie X, Wu Y, Wang Q, Zhao Y, Chen G, Xiang B. A randomized trial of pneumatic reduction versus hydrostatic reduction for in those structures that are less rigid and more impacted by respiration, heartbeat, and surgical perturbation has been called into question. Recently, LARN has been successfully applied in a less rigid surgical navigation of the liver (10).

Similar to the liver, the spatial relationships between structures in the digestive tract are inconsistent, and deformations are relatively common. This makes AR-assisted procedures more challenging. Resultantly, there is less data available on the value and efficacy of AR in laparoscopic surgeries of the gastrointestinal tract (14). Nonetheless, the success of the procedure in the stereoscopic laparoscopic hepatectomy of liver cancer in the new methodology from Zhang et al. (10) suggests that these techniques may show promise in gastrointestinal surgeries, like intussusception reductions.

\section{CONCLUSION}

In the future, the robustness of LARN needs to be further assessed in all surgical procedures, especially digestive organs like the intestines. The following would be required for eventual integration into regular practice for laparoscopic intussusception surgeries: LARN needs to (1) consistently show positive outcomes in the GI tract and (2) enhance enough important information in the visual field of the intestines to outweigh the information that is inevitably occluded (14).

\section{AUTHOR CONTRIBUTIONS}

All authors listed have made a substantial, direct and intellectual contribution to the work, and approved it for publication.

\section{FUNDING}

SS and FQ are recipients of the Sarah Morrison Research Award at the University of Missouri-Kansas City School of Medicine. intussusception in pediatric patients. J Pediatr Surg. (2018) 53:1464-8. doi: 10.1016/j.jpedsurg.2017.08.005

7. Chew R, Ditchfield M, Paul E, Goergen SK. Comparison of safety and efficacy of image-guided enema reduction techniques for paediatric intussusception: A review of the literature. J Med Imaging Radiat Oncol. (2017) 61:711-7. doi: 10.1111/1754-9485.12601

8. Tafner E, Tafner P, Mittledorf C, Pinhata J, Silva A, Pilli S, et al. Potential of colonoscopy as a treatment for intussusception in children. Endoscopy Int Open. (2017) 5:E1116-8. doi: 10.1055/s-0043-117950

9. Park JK, Kwon TH, Kim HK, Park JB, Kim K, Suh JI. Adult intussusception caused by an appendiceal mucocele and reduced by colonoscopy. Clin Endosc. (2011) 44:133. doi: 10.5946/ce.2011.44.2.133

10. Zhang W, Zhu W, Yang J, Xiang N, Zeng N, Hu H, et al. Augmented reality navigation for stereoscopic laparoscopic anatomical hepatectomy of primary liver cancer: preliminary experience. Front Oncol. (2021) 11:663236. doi: $10.3389 /$ fonc.2021.663236

11. Cabrilo I, Schaller K, Bijlenga P. Augmented reality-assisted bypass surgery: embracing minimal invasiveness. World Neurosurg. (2015) 83:596-602. doi: 10.1016/j.wneu.2014.12.020

12. Liu WP, Richmon JD, Sorger JM, Azizian M, Taylor RH. Augmented reality and cone beam CT guidance for transoral robotic surgery. J Robot Surg. (2015) 9:223-33. doi: 10.1007/s11701-015-0520-5 
13. Teber D, Guven S, Simpfendörfer T, Baumhauer M, Güven EO, Yencilek F, et al. Augmented reality: a new tool to improve surgical accuracy during laparoscopic partial nephrectomy? Preliminary in vitro and in vivo results. Eur Urol. (2009) 56:332-8. doi: 10.1016/j.eururao.2009. 05.017

14. Bernhardt S, Nicolau SA, Soler L, Doignon C. The status of augmented reality in laparoscopic surgery as of 2016. Med Image Anal. (2017) 37:66-90. doi: 10.1016/j.media.2017. 01.007

Conflict of Interest: The authors declare that the research was conducted in the absence of any commercial or financial relationships that could be construed as a potential conflict of interest.
Publisher's Note: All claims expressed in this article are solely those of the authors and do not necessarily represent those of their affiliated organizations, or those of the publisher, the editors and the reviewers. Any product that may be evaluated in this article, or claim that may be made by its manufacturer, is not guaranteed or endorsed by the publisher.

Copyright (c) 2021 Singh, Borthwick and Qureshi. This is an open-access article distributed under the terms of the Creative Commons Attribution License (CC BY). The use, distribution or reproduction in other forums is permitted, provided the original author(s) and the copyright owner(s) are credited and that the original publication in this journal is cited, in accordance with accepted academic practice. No use, distribution or reproduction is permitted which does not comply with these terms. 